\title{
Einführung: Strom à la carte
}

\section{K. Dorfmeister OVE}

Online publiziert am 20. November 2017

(C) Springer-Verlag GmbH Austria, ein Teil von Springer Nature 2017

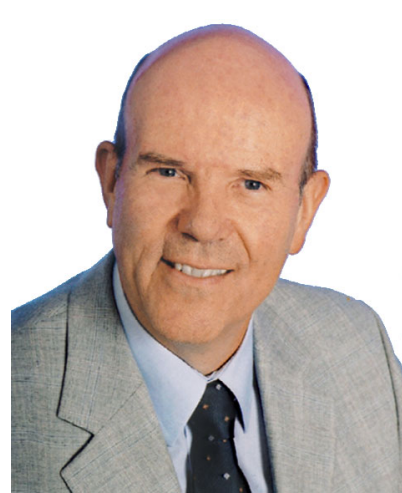

Dipl.-Ing. Dr. Karl Dorfmeister
Strom aus der Steckdose, am besten nach Wahl wie aus einer Menükarte - gibt es das wirklich, und steht Strom so allen Anwendern nach Belieben zur Verfügung?

Diese Frage stellt sich zwangsläufig, verfolgt man die Medienmeldungen zum Thema elektrischer Strom aus den unterschiedlichsten Betrachtungsrichtungen. Es lässt sich aber auch der Eindruck nicht verwehren, dass das Thema "Strom" je nach Stimmung und Bedarf in der Öffentlichkeit aufgegriffen und entsprechend breitgetreten wird.

Dabei dürfte im Bewusstsein aller noch nicht so richtig verankert sein, dass die elektrische Energie im täglichen Leben einen ganz wichtigen Stellenwert eingenommen hat, um nicht sogar von einer Lebensnotwendigkeit zu sprechen. Die Selbstverständlichkeit des "Stroms aus der Steckdose" wird einfach hingenommen, als ob dies natürlich so zu sein hat. Vergessen wird dabei aber vollkommen, dass für diese „natürliche Energieversorgung" das Zusammenwirken vieler Einzelkomponenten erforderlich ist, das nur in ganz geringem Ausmaß vorgegebenen Regeln folgt, ansonsten jedoch ausschließlich physikalischen Gesetzen gehorcht. Die Technik kann sich bestenfalls der Physik bedienen und diese für ihre Erfordernisse „ausnutzen”, um so die Wünsche der Anwender erfüllen zu können.

Da die Anforderungen der verschiedenen Stromabnehmer sehr unterschiedlich sind, hat sich die Österreichische Gesellschaft für Energietechnik in ihrem 50. Bestandsjahr mit ihrer 55. Fachtagung unter dem Titel "Strom à la carte - Vielfalt der Anwendungen und Lösungen" dieser Thematik angenommen.

Es ist gelungen, aus den wesentlichen Gruppen der Stromkunden kompetente sowie einflussreiche Vertreter zu finden, die basierend auf ihren Erfordernissen die Wünsche an die Stromversorgung aufzeigen. Wie diesen Anforderungen entsprochen wird bzw. noch nachgekommen werden kann, zeigen hochrangige Experten der Elektrizitätswirtschaft, der Elektroindustrie sowie aus Lehre und Forschung auf.

Die OGE bedankt sich besonders bei folgenden Sponsoren der Fachtagung für ihre tatkräftige Unterstützung: $A B B$ AG, ANDRITZ HYDRO GmbH, Austrian Power Grid AG, Bundesministerium für Verkehr, Innovation und Technologie, Energienetze Steiermark GmbH, Österreichische Forschungsförderungsgesellschaft $\mathrm{GmbH}$, PHOENIX CONTACT GmbH, Pöyry Energy GmbH, Salzburg AG (Hauptsponsor), Schneider Electric Austria Ges.m.b.H., SIEMENS AG Österreich, Sprecher Automation $\mathrm{GmbH}$, TIWAG-Tiroler Wasserkraft AG und Wels Strom GmbH.
Einführung zur 55. Fachtagung der Österreichischen Gesellschaft für Energietechnik (OGE) im OVE, die am 12. und 13. Oktober 2017 in Salzburg stattfand.

Karl Dorfmeister Tagungsleiter und Geschäftsführer der Österreichischen Gesellschaft für Energietechnik (OGE) im OVE.

Dorfmeister, Karl, Österreichische Gesellschaft für Energietechnik (OGE) im OVE, Eschenbachgasse 9, 1010 Wien, Österreich (E-Mail: k.dorfmeister@ove.at) 